\title{
A Hybrid Communication Pattern in Brain Structural Network Revealed by Evolutionary Computation
}

\author{
Quanmin Liang, Junji Ma, Xitian Chen, Zhengjia Dai ${ }^{*}$, Ying Lin ${ }^{* \dagger}$ \\ Department of Psychology, Sun Yat-sen University, Guangzhou, China, 510006
}

* Corresponding Authors:

Zhengjia Dai, Ph.D., Department of Psychology, Sun Yat-sen University, Guangzhou, China, 510006. Email: daizhengj@mail.sysu.edu.cn

Ying Lin, Ph.D., Department of Psychology, Sun Yat-sen University, Guangzhou, China,

510006. Email: linying23@mail.sysu.edu.cn

$\dagger$ Lead Contact 


\section{Summary}

The human brain functional connectivity network (FCN) is constrained and shaped by the information communication processes in the structural connectivity network ( $\mathrm{SCN})$. The underlying communication model thus becomes a critical issue for understanding structure-function coupling in the human brain. A number of communication models featuring different point-to-point routing strategies have been proposed, with shortest path (SP), diffusion (DIF), and navigation (NAV) as the typical, respectively requiring network global knowledge, local knowledge, and their combination for path seeking. Yet these models all assumed the entire brain to use a uniform routing strategy, which ignored lumping evidence supporting the wide variety of brain regions in both terms of biological substrates and functional exhibitions. In this study, we developed a novel communication model that allowed each brain region to choose a routing strategy from SP, DIF, and NAV independently. A genetic algorithm was designed to uncover the underlying region-wise hybrid routing strategy (namely HYB) for maximizing the structure-function coupling. The HYB-based model outperformed the three typical models in terms of predicting FCN and supporting robust communication. In HYB, brain regions in lower-order functional modules inclined to choose the routing strategies requiring more global knowledge, while those in higher-order functional components preferred to choose DIF. Additionally, compared to regions using SP and NAV, regions using DIF had denser structural connections, participated in more functional modules, but were less dominant within them. Together, our findings revealed and evidenced the possibility and advantages of hybrid routing underpinning efficient SCN communication.

Keywords: brain connectome, communication, routing, structure-function coupling, evolutionary computation 


\section{Introduction}

With the advance of magnetic resonance imaging (MRI) technology, the human neural system can be characterized as a complex network where gray matter regions (nodes) are interconnected by white-matter projections (links), namely the brain structural connectivity network (SCN) [1-3]. Previous studies have shown that the SCN possesses multiple non-random topological properties, including small-worldness [4, 5], modular structure [6], and a highly interconnected rich-club organization $[7,8]$, which facilitates communication among brain regions [9]. It has also been recognized that communication paths in the SCN (i.e., transmission routes of neural signals along white-matter pathways) are crucial for understanding how the functional connectivity network (FCN), where brain regions are interconnected by functional connectivity (i.e., temporal correlations), is constrained and shaped by the SCN $[10,11]$. A series of models centered around communication paths have been developed to predict FCN from SCN (e.g., shortest-path-based global efficiency, or multi-path-based communicability) [12-15]. However, although the significance of SCN communication has been widely admitted and demonstrated, the underlying routing strategy, i.e., how neural signals are routed through the white-matter pathways, remains an open challenge $[3,16]$.

Initially, exploration of the communication models in the SCN was mainly based on the idea of optimal routing. That is, neural signals are considered transmitted through the shortest paths between brain regions $[13,17,18]$. The shortest-path routing is indeed fast and straightforward, effectively reducing the risk of signal loss [19]. However, the deficiency is also obvious: it requires every region to obtain information about the global network topology [20, 21], which induces heavy informational costs [22]. In addition, the shortest-path routing utilizes only a small portion of links in the SCN, resulting in a disproportionately large information flow over a small number of connections [23]. The 
network is thus prone to information congestion and targeted attack [24, 25]. In contrast to the idea of optimal routing, some researchers proposed communication models in the SCN based on the concept of random walking [21]. Since random walkers rely purely on local topological information (e.g., connection strength) [22], the random-walking-based routing strategies remove the premise that all elements in the network need to know the global network topology and thus relieve the burden of informational cost. It also helps reduce the information congestion phenomenon and improve the network robustness by balancing information flow on network connections. However, previous studies have shown that they may lead to longer transmission routes and thus slow down the transmission speed, resulting in inefficient communication among brain regions [21].

Since neither optimal routing nor random walking could fully support or explain the communication patterns observed in the human brain, the idea of hybridizing local and global information was proposed, leading to a navigation routing strategy greedily guided by local adjacency and global spatial positions $[9,26]$. Recently, it was further suggested that instead of fixed hybridization, the routing strategy in the human brain is more likely on a spectrum spanning from pure utilization of global information (optimal routing) to pure utilization of local information (random walking) [22]. Research efforts have been committed to striking an ideal point on this spectrum such that the resulting communication model can hybridize the strengths of optimal routing and random walking. Notably, despite the hybrid usage of global and local information, these communication models still inherited a common premise: all brain regions use the same routing strategy. That is, all the brain regions share the same utilization pattern of local and/or global information. This premise, although significantly simplifies the communication dynamics in the brain network, is unlikely to be realistic, giving that there has been convergent evidence supporting the distinctive roles of brain regions in communication. For example, some tightly connected regions form a 'rich club' 
that makes an important contribution to inter-regional signaling [7, 27]. Efficient routing could also be achieved when only a limited number of regions 'know' the global information [22]. In addition, it has been shown that brain networks are resilient to lesions due to the involvement of alternative communication paths, implying the possibility of adaptively switching regional roles in communication $[28,29]$. It is thus reasonable to conjecture that brain regions could adopt different information routing strategies.

Relaxing the premise of uniform routing, this study proposed a novel communication model underpinned by a region-wise hybrid routing framework that allowed brain regions to select routing strategies from three representatives: shortest path (pure global information), random diffusion (pure local information), and navigation (mixed local-global information). Notably, this hybrid framework tolerated the extreme situation that all brain regions select the same routing strategy and allowed the hybridization to degenerate into one of the three classic routing strategies. Relying on the imaging data from a cohort of 88 healthy adults, a genetic algorithm [30], which is a model-free metaheuristics in the domain of evolutionary computation well-known for its global search capability, was designed to identify the region-wise routing hybridization that could best explain the variation in functional connectivity. The advantages of the obtained routing hybridization over the three representatives were then examined from the perspectives of how well it supported the functional connectivity network (FCN) and how robust it made the communication process in the SCN. The plausibility of the regional choices on the routing strategies was also analyzed concerning their topological roles in brain networks.

\section{Results}

In the current study, the core idea of the proposed communication model lies in the region-wise hybrid routing framework, i.e., each brain region determines the next stop to 
transmit neural signals based on its own choice from three potential routing strategies: shortest path (SP), navigation (NAV), and random diffusion (DIF). Given that the brain was parcellated into $N$ regions (herein $N=90$, see the method section), there would be $3^{N}$ possible combinations, making the search for an effective hybrid routing strategy intractable. To address this issue, we proposed to turn the search into a combinatorial optimization problem, with the objective defined as maximizing the correlation between structural routing efficiency and empirical functional connectivity strength over all region pairs [15]. Due to the huge search space and the high computational intensity for evaluating a potential combination, we designed a meta-heuristic, the genetic algorithm (GA) [21, 30-32], to find a near-optimal hybrid routing strategy (denoted as 'HYB') in acceptable computational time. The effectiveness of HYB was first validated through comparison with the random baseline and reproducibility analyses. Then the composition of HYB was mapped on the brain and investigated in relation to functional modules. Comparing with SP, NAV, and DIF, the performance of HYB in supporting functional connectivity, balancing information flow, and resisting targeted lesion was examined to illustrate its advantage. Finally, the plausibility analyses regarding the HYB were performed to highlight its rationality and implications. For more details about the above analysis methods, refer to the method section.

\section{Discussion}

This study proposed a communication model for the human brain structural connectivity network $(\mathrm{SCN})$ based on the idea of region-wise hybrid routing, which assumed brain regions to adopt different routing strategies for neural signal transmission. The hybrid routing strategy was approximated through a genetic algorithm (GA) that aimed at maximizing the coupling between structural routing efficiency and functional connectivity. Based on the obtained solution (namely 'HYB'), our main findings were as follows. Firstly, the regions 
adopting different strategies did not randomly scatter over the brain, but showed a specific clustering pattern that partially complied with the partition of functional modules. Secondly, the proposed HYB strategy was advantageous over the other three strategies both in terms of capturing the functional connectivity network $(\mathrm{FCN})$ and supporting robust communication in the SCN. Thirdly, by analyzing the regional choices of routing strategies, we found that brain regions in lower-order functional modules (e.g., VIS and SMN) inclined to choose the routing strategies requiring more global information (i.e., SP and NAV), while those in higher-order functional components were more likely to choose the DIF strategy. In addition, compared to the brain regions using SP and NAV, the brain regions using the DIF strategy had larger degrees in the SCN and higher Pc in the FCN, but lower degree Z-score within functional modules. In conclusion, our results supported that the availability of global topological information distributed with regional heterogeneity over the brain, and the resulting communication process facilitated integration and segregation of information and thus the formation of functional modules.

\section{Revealing Regionally Heterogeneous Distribution of Global Topological Information}

It has been widely admitted that global topological information plays an important role in the communication processes of complex systems [20], and its availability can lead to different communication models from random diffusion to optimal routing [22]. The SCN of human brain, which possess the general properties of complex systems [13, 38, 39], is thus also considered to utilize global topological information for achieving efficient communication.

However, most previous studies on brain communication presumed that all the brain regions used the same communication strategy, implicitly assuming that the whole brain has equivalent availability of global information $[9,18]$. In the current study, we challenged the above assumption by proposing a region-wise hybrid routing framework that allowed brain regions to adopt different routing strategies requesting different levels of global information. 
Such difference could not only influence the communication efficiency of individual brain regions, but also shape the global dynamics of information flow in the brain and thus contribute to our understanding towards brain functional integration and segregation.

Our results showed that the region-wise hybrid routing framework could better explain the FC variation from the global level, modular level to regional level, suggesting that the regionally heterogeneous distribution of global information availability may be a more realistic assumption. We also discovered that such a regionally heterogeneous distribution was a stable and organized property widely existing in the brain, but meanwhile showing certain functional-module specificity. In the primary cortices (i.e., VIS and SMN), most brain regions used SP and NAV for routing, showing higher availability of global information. The proportion of brain regions using DIF markedly rose in the association cortices (e.g., DAN, VAN, and DMN in particular), indicating decrease in global information availability. The association cortices encapsulate more advanced functions such as cognition, attention, etc., and compose a core functional module, whereas the primary cortices participate in more direct perceptual tasks, such as vision, motor-auditory, etc. [40-42]. Our results shed light on a possible information communication mechanism behind these functions. That is, direct perceptual functions prefer straightforward and rapid information transfer, whilst advanced cognitive functions require the involvement of multiple modules and therefore prefer (or allow) decentralized and slower information transfer. Indeed, this regional heterogeneity was also observed by previous structure-function coupling studies [43-46]. Specifically, the human brain structure and function tightly coupled in the primary cortices but decoupled in the association cortices. The higher availability of global information of primary cortices may allow optimal and unique transmission routes, which effectively reduced the risk of signal loss and may further result in tight structure-function coupling. Meanwhile, the lower availability of global information of association cortices may lead to longer and more diverse 
transmission routes, which may increase the uncertainty of information transmission, and eventually lead to decoupling of structure and function. In addition, the association cortices act as a structural and functional core organization of human brain and had disproportionately high number of connections to other regions [36]. The lower availability of global information of these regions could effectively reduce the burden of informational cost. Taken together, these findings highlight the potential of the region-wise hybrid routing strategy to support large-scale neural signaling transfer in the human brain.

\section{Supporting Robust Communication in SCN}

Robustness is an important aspect in addition to efficiency that drives the communication processes in the SCN $[39,47,48]$. Routing strategies should therefore be able to avoid signaling bottleneck and promote network resilience [23, 24, 28]. We compared the robustness of SCN communication under the proposed HYB strategy and the three classic routing strategies through analyses of nodewise/edgewise betweenness centrality $(\mathrm{Bc})$ and a lesion simulation of targeted attack.

The results regarding $\mathrm{Bc}$ showed that the proposed HYB strategy was second to the best DIF strategy in balancing the load of information flow over the SCN, implying that HYB may also be able to relieve information congestion like DIF did [16, 49]. In contrast, the signaling procedures of the SP and NAV strategies unfolded mainly along a small fraction of fiber tracts, resulting in a high risk of encountering signaling bottleneck $[9,15,23]$. As the classic DIF strategy, the load balancing ability of HYB is mainly attributable to its recruitment of different alternative pathways between region pairs $[29,50]$. Notably, with only $40 \%$ of the brain regions using DIF, the HYB strategy achieved pathway diversity sufficient to markedly reduce unreasonable load allocation, which underscores the advantage and necessity of region-wise hybrid routing. In canonical and complex networks, balancing load provides a better basis to counteract attack [51-53]. The same pattern was also observed 
in our results of lesion simulation. Importantly, the HYB strategy entailed the lowest network vulnerability at the early stage of lesion, implying that it was the most resilient to attack at the highly connected hubs and thus provided a stronger neural substrate for the plasticity of brain connectome [54-56]. Combining with our findings that regions adopting DIF had larger structural degrees, we conjectured that the efficiency decay shrunk probably because the highly connected hubs mainly routed in a decentralized and random manner, which improves resilience in certain sacrifice of efficiency $[21,23,57,58]$. Further, the comparison of the initial network efficiency among the four strategies revealed that the HYB strategy could still approximate the optimal efficiency achieved by SP, supporting the notion that efficient neural communication is not necessarily constrained to centralized optimal routing [9, 12]. Taken the above results together, we recapitulated that the proposed HYB strategy managed to simultaneously address the shortcoming of SP and NAV in terms of robustness and the shortcoming of DIF in terms of efficiency $[9,16,58]$. Overall, it could be stated that the HYB strategy outperformed the other three strategies in terms of striking a balance between communication efficiency and resilience. Such an advantage is probably attributed to the assignment of routing strategies with different strengths and weakness to specific brain regions.

\section{Plausibility behind the Regional Choices of Routing Strategies}

Our analyses about the relationship between the regional topological characteristics and their choices of routing strategies provided some plausible explanations for the HYB. First, the regions using DIF were found to have significantly more structural links than those using SP and NAV. The reasons could be two-fold. On the one hand, previous studies have shown that high-degree nodes consume higher informational cost in communication [22]. Using the DIF strategy that requires no global information thus effectively reduces the informational cost of the whole network. On the other hand, high-degree nodes usually function as hubs in the 
network $[7,59]$ and carry out huge information transfer tasks. Adopting the DIF strategy thus enables a more balanced utilization of the edges connected to them compared to the SP and NAV strategies, leading to a more robust communication process. Second, we also found that the regional choice of routing strategy was related to its role within and across functional modules. In detail, our findings showed that the peripheral nodes, which are mainly connected to their own modules $(\mathrm{Z} \leq 0.9, \mathrm{Pc} \leq 0.3)$, mostly adopted the NAV strategies, while provincial hubs, which act as messaging hubs within modules $(\mathrm{Z}>0.9, \mathrm{Pc} \leq 0.3)$, adopted only SP and NAV strategies. In addition, connector hubs, which act as messaging hubs for the whole network $(\mathrm{Z}>0.9, \mathrm{Pc}>0.3)$, mainly adopted the SP strategy. From the perspective of information aggregation and dispersion, the SP and NAV strategies enable fast signal transmission to targets and thus help aggregate information efficiently $[22,26]$. In particular, the NAV strategy, a greedy routing algorithm based on spatial distance, reduces the use of global information while maintaining a high efficiency of information transfer in local area [9, 26], making it more suitable for information aggregation within modules. Conversely, the DIF strategy promotes information dispersion in a random walking manner [16, 49]. We noted that peripheral nodes and provincial hubs, which were in charge of intra-module information transfer, mainly adopted the SP and NAV strategies, which can quickly facilitate intra-module information aggregation and reduce the use of global information. In contrast, as for both satellite connectors $(\mathrm{Z} \leq 0.9, \mathrm{Pc}>0.3)$ and connector hubs that were in charge of inter-module information transfer, DIF accounted for a high proportion, which contributes to a certain extent to information dispersion between modules and also reduces the information transfer burden of connectors. This may provide a potential routing basis for the formation of functional modules. i.e., the information transfer within modules is fast and direct, while the information between modules is relatively slow and dispersed.

\section{Limitations and Future Directions}


The current work had several limitations that should be considered. First, the current study relaxed the assumption that all brain regions required the same amount of global information of the brain. Instead, we allowed each brain region to decide how much global information was needed. However, the biological mechanism of how to access global information remains unclear [9]. Future studies may consider more realistic constraints about accessing global information, including the accessibility and the assessing cost, etc. Second, the brain networks in the current study were constructed by widely accepted methods, with nodes defined using the AAL90 template, structural links defined by the fiber density obtained from DWI data, and functional links defined by the Pearson correlations derived from R-fMRI. Such typical construction methods were ideal for the initial attempt of proving our idea of region-wise hybrid routing in the SCN. However, it has been proven that different construction methods can influence the topology of brain networks and thus subsequent analyses $[60,61]$. In the future, our findings should be validated on more brain networks constructed by different methods based on third-party datasets. Third, the focus of our work was on the communication procedure at the resting state. This is because the resting-state FC pattern has been found to be an intrinsic brain organization state and associated with a variety of cognitive tasks [62-64]. It would be interesting to investigate whether and how our findings and approaches can be used to identify the routing strategies in different cognitive tasks. In addition, future research should also consider further extending the proposed GA as well as other evolutionary algorithms, which have been proven to be powerful for solving a wide range of routing problems in many fields [65-67], for better addressing the routing strategies underpinning the communication processes in the human brain.

In conclusion, our proposed approach has great potential to explore brain network communication processes and can be used to investigate the real way of information exchange in complex systems and their subsystems. We have also discovered that global 
bioRxiv preprint doi: https://doi.org/10.1101/2021.11.25.469862; this version posted November 25, 2021. The copyright holder for this preprint (which was not certified by peer review) is the author/funder, who has granted bioRxiv a license to display the preprint in perpetuity. It is made available under aCC-BY-NC-ND 4.0 International license.

information availability may be non-uniformly distributed throughout the brain, providing a new perspective on the formation of functional modules and the relationship between brain structure and function. 


\section{Materials and Methods}

\section{Neuroimaging Participants}

We recruited 93 participants from the South China Normal University, Guangzhou, China. All participants were right-handed, had a normal or corrected vision, no neurological or psychiatric disorders, and no sensorimotor or cognitive impairment. Five participants were excluded due to excessive head motion during scanning (head rotation $>2^{\circ}$ or translation $>2$ $\mathrm{mm}$, see Image Preprocessing for details), resulting in a total of 88 participants (mean age $=$ $18.98 \pm 1.08$ years old; 60 females).

\section{MRI Data Acquisition}

All participants were scanned on a Siemens 3.0T MRI scanner (Siemens, Erlangen, Germany) with an 8-channel head coil at the South China Normal University. Headphones and foam pads were used during the scan to avoid interference from scanner noise and to reduce head movements. Before the scan, participants were instructed to keep their eyes closed, stay awake without thinking anything and keep their head fixed during the scan. The structural T1 weighted images were collected using magnetization prepared by rapid gradient echo sequence with the following parameters: $\mathrm{TR}=1900 \mathrm{~ms}, \mathrm{TE}=2.52 \mathrm{~ms}, \mathrm{FA}=9^{\circ}, \mathrm{FOV}=256 \times$ $256 \mathrm{~mm}^{2}$, inversion time $=900 \mathrm{~ms}$, matrix $=256 \times 256$, slices $=176$, slice thickness $=1 \mathrm{~mm}$, and voxel size $=1 \times 1 \times 1 \mathrm{~mm}^{3}$. The diffusion-weighted imaging $(\mathrm{DWI})$ data were acquired with the following parameters: repetition time $(\mathrm{TR})=10000 \mathrm{~ms}$, echo time $(\mathrm{TE})=90 \mathrm{~ms}$, matrix $=128 \times 128$, field of view $(\mathrm{FOV})=256 \times 256 \mathrm{~mm}^{2}$, flip angle $(\mathrm{FA})=90^{\circ}$, slice thickness $=2 \mathrm{~mm}$ without gap. The diffusion sensitizing gradients were applied along 64 non-collinear directions $\left(b=1000 \mathrm{~s} / \mathrm{mm}^{2}\right)$, together with one acquisition without diffusion weighting $\left(b=0 \mathrm{~s} / \mathrm{mm}^{2}\right)$. Resting-state functional MRI (R-fMRI) data were imaged using an echo-planar imaging sequence with the following parameters: TR $=2000 \mathrm{~ms}, \mathrm{TE}=30 \mathrm{~ms}, \mathrm{FA}$ $=90^{\circ}, \mathrm{FOV}=224 \times 224 \mathrm{~mm}^{2}$, slices $=32$, matrix $=64 \times 64$, slice thickness $=3.5 \mathrm{~mm}$, and 
voxel size $=3.5 \times 3.5 \times 3.5 \mathrm{~mm}^{3}$. Totally 240 volumes of $\mathrm{R}$-fMRI data were collected in the scan. After scanning, all participants confirmed that they had stayed awake during the scan.

\section{Image Preprocessing}

The DWI data were preprocessed using the PANDA toolbox [70]. The preprocessing process included brain extraction, correction for eddy-current distortion, head motion, and b-matrix [72]. The diffusion tensor and the fractional anisotropy (FA) were calculated. In addition, to obtain a white matter (WM) binary mask in the native diffusion space, which was used to provide a constraint mask for the subsequent WM tractography, the T1-weighted images were first manually positioned onto the AC-PC line and then segmented using SPM8 software (http://www.fl.ion.ucl.ac. uk/spm). The resulting WM binarization mask (with WM probability threshold >0) in the T1 space was calibrated to the diffusion space using the inverse transformation matrix estimated during the co-registration of the FA image to T1.

The R-fMRI data were preprocessed using the Data Processing Assistant for Resting-State fMRI (DPARSF) [69] and the SPM8 toolbox. The R-fMRI preprocessing steps were as follows: (1) deleted of the first ten time points, followed by time layer correction (slice timing) on the remaining 230 volumes; (2) head motion correction and five participants were excluded due to excessive head motion (head rotation $>2^{\circ}$ or translation $>2 \mathrm{~mm}$ ); (3) segmentation of structural images into gray matter (GM), white matter (WM), and the cerebrospinal fluid (CSF) using a uniform segmentation algorithm [73]; (4) normalized the motion-corrected functional image to the Montreal Neurological Institute (MNI) space using the normalization parameters estimated during unified segmentation and resampled to 3-mm isotropic voxels; (5) Gaussian smoothing (FHWM $=4 \mathrm{~mm} \times 4 \mathrm{~mm} \times 4 \mathrm{~mm}$ ) was performed; and (6) linear trends were removed and regressed on extraneous variables (Friston 24 head motion parameters, WM and CSF signals); (7) Band-pass filtering with a frequency of 0.01-0.08 Hz. 


\section{Construction of Structural Connectivity Network}

Nodes of the SCN were defined by the Automated Anatomical Labeling parcellation scheme (AAL90, 90 nodes/regions in total excluding the cerebellum) [74]. This atlas was originally defined in the standard Montreal Neurological Institute (MNI) space and transformed into a native diffusion space for each participant following the typical procedure of previous studies [75]. Specifically, individual FA images in the native diffusion space were first co-registered with the T1-weighted images. Then the T1-weighted images were normalized to the ICBM152 T1 template in the MNI space. Finally, the two transformation matrices were inverted so that each participant's AAL atlas was calibrated from MNI space to the native diffusion space.

For defining edges of the SCN, we performed the deterministic tractography based on fiber assignment by continuous tracking (FACT) using the Diffusion Toolkit (http://trackvis.org) [76]. The tracking procedure started from seed at the center of each voxel with an FA value greater than 0.2 in the WM mask and terminated when reaching a voxel with the turning angle greater than $45^{\circ}$, or with an FA value less than 0.2 , or located out of the WM mask. We then used the fiber density between each node pair (reciprocal of fiber length per unit surface) to weight the edges in the SCN [77], where larger weights implied stronger structure connectivity. The group-level SCN was further defined by averaging the weight of each edge over all the individual SCNs after filtering out the edges present in less than $25 \%$ of the participants [78]. Finally, to facilitate estimation of the transmission cost, we transformed the edge weight into edge length by $l_{i j}=-\ln \left(w_{i j}\right)$, where $w_{i j}$ was the weight between two nodes $i$ and $j(i, j=1,2, \ldots, 90, i \neq j)$ [49]. A longer edge implied a larger transmission cost. Using the edge lengths as entries, a 90 by 90 symmetric weighted matrix can then be obtained to represent the group-level SCN over the 88 participants.

\section{$\underline{\text { Construction of Functional Brain Connectivity }}$}


Consistent with the SCN, the functional connectivity network (FCN) was also composed of 90 nodes derived by parcellating the brain gray matter into 90 regions according to the AAL template. Then for each participant, the time series of all voxels in each brain region were averaged and the Pearson correlation coefficient was calculated between the average time series of each brain region pair as the corresponding functional connectivity strength. A 90 by 90 symmetric weighted matrix was thus obtained to represent the FCN of each participant. Finally, the individual FCN matrices of all the 88 participants were averaged to obtain the group-level FCN matrix, which was used in subsequent analyses.

\section{Validation Analyses}

We further validated the proposed communication model based on region-wise hybrid routing on an independent dataset (denoted as 'BNU'), which is a subset of the Connectivity-based Brain Imaging Research Database (C-BIRD) at Beijing Normal University and can be acquired from the publicly available data of Consortium for Reliability and Reproducibility (CoRR) (http://fcon_1000.projects. nitrc.org/indi/CoRR/html/bnu_1.html) [68] The dataset consisted of two MRI scan sessions from 57 healthy adults (male: 30; age: 19-30 years), completed over a time interval of approximately 6 weeks (40.94 \pm 4.51 days). In particular, the first scan session included two R-fMRI scans, T1, T2, and Diffusion-tensor images (DTI). Only the data of the first R-fMRI scan was used in the validation study. All participants were right-handed and had no history of neurological or psychiatric disorders. Written informed consent was obtained from each participant, and data collection was approved by the Institutional Review Board of the State Key Laboratory of Cognitive Neuroscience and Learning, Beijing Normal University. The BNU dataset was preprocessed using the same pipeline as in the main study. Two participants were excluded, one for excessive head motion (translation $>2 \mathrm{~mm}$ or rotation $>2^{\circ}$ ) and the other one for missing slices in R-fMRI data. The 
proposed GA was then applied to the group-average SCN of the 55 participants, and the resulting hybrid routing strategy (denoted as 'B-HYB') was analyzed to examine whether our main findings were reproducible in the following three aspects: advantage in capturing the FCN, support to robust communication, and plausibility behind the regional choices of different routing strategies. 


\section{Reference}

1. Sporns, O., Tononi, G., and Kotter, R. (2005). The human connectome: A structural description of the human brain. PLoS Comput Biol 1, e42.

2. Bassett, D.S., and Sporns, O. (2017). Network neuroscience. Nature Neuroscience 20, 353-364.

3. Fornito, A., Zalesky, A., and Bullmore, E.T. (2016). Fundamentals of Brain Network Analysis. sociological quarterly.

4. Bassett, D.S., and Bullmore, E. (2006). Small-World Brain Networks. The Neuroscientist 12, 512-523.

5. Watts, D.J., and Strogatz, S.H. (1998). Collective dynamics of 'small-world' networks. Nature 393, 440-442.

6. Sporns, O., and Betzel, R.F. (2016). Modular Brain Networks. Annual Review of Psychology 67, 613-640.

7. van den Heuvel, M.P., Kahn, R.S., Goni, J., and Sporns, O. (2012). High-cost, high-capacity backbone for global brain communication. Proc Natl Acad Sci U S A 109, 11372-11377.

8. Van, d.H., M. P., and Sporns, O. (2011). Rich-Club Organization of the Human Connectome. Journal of Neuroscience 31, 15775-15786.

9. Seguin, C., van den Heuvel, M.P., and Zalesky, A. (2018). Navigation of brain networks. Proceedings of the National Academy of Sciences 115, 6297-6302.

10. Cole, M.W., Ito, T., Bassett, D.S., and Schultz, D.H. (2016). Activity flow over resting-state networks shapes cognitive task activations. other.

11. Medaglia, J.D., Lynall, M.-E., and Bassett, D.S. (2015). Cognitive Network Neuroscience. Journal of Cognitive Neuroscience 27, 1471-1491.

12. Goni, J., van den Heuvel, M.P., Avena-Koenigsberger, A., Velez de Mendizabal, N., Betzel, R.F., Griffa, A., Hagmann, P., Corominas-Murtra, B., Thiran, J.P., and Sporns, O. (2014). Resting-brain functional connectivity predicted by analytic measures of network communication. Proc Natl Acad Sci U S A 111, 833-838.

13. Rubinov, M., and Sporns, O. (2010). Complex network measures of brain connectivity: uses and interpretations. Neuroimage 52, 1059-1069.

14. Andreotti, J., Jann, K., Melie-Garcia, L., Giezendanner, S., Abela, E., Wiest, R., Dierks, T., and Federspiel, A. (2014). Validation of network communicability metrics for the analysis of brain structural networks. In PloS one, Volume 9. p. e115503.

15. Seguin, C., Tian, Y., and Zalesky, A. (2020). Network communication models improve the behavioral and functional predictive utility of the human structural connectome. Netw Neurosci 4, 980-1006.

16. Avena-Koenigsberger, A., Misic, B., and Sporns, O. (2017). Communication dynamics in complex brain networks. Nat Rev Neurosci 19, 17-33.

17. Kaiser, M. (2006). Nonoptimal Component Placement, but Short Processing Paths, due to Long-Distance Projections in Neural Systems. PLOS Computational Biology 2.

18. Bullmore, E., and Sporns, O. (2009). Complex brain networks: Graph theoretical analysis of structural and functional systems (Nature Reviews Neuroscience (2009) 10, (186-198)). Nature reviews Neuroscience 10, 186-198.

19. Crucitti, P., Latora, V., Marchiori, M., Rapisarda, and A. (2003). Efficiency of 
scale-free networks: error and attack tolerance. PHYSICA A.

20. Boccaletti, S., Latora, V., Moreno, Y., Chavez, M., and Hwang, D.U. (2006). Complex networks: Structure and dynamics. Physics Reports 424, 175-308.

21. Goni, J., Avena-Koenigsberger, A., Velez de Mendizabal, N., van den Heuvel, M.P., Betzel, R.F., and Sporns, O. (2013). Exploring the morphospace of communication efficiency in complex networks. PLoS One 8, e58070.

22. Avena-Koenigsberger, A., Yan, X., Kolchinsky, A., van den Heuvel, M.P., Hagmann, P., and Sporns, O. (2019). A spectrum of routing strategies for brain networks. PLoS Comput Biol 15, e1006833.

23. Avena-Koenigsberger, A., Misic, B., Hawkins, R.X., Griffa, A., Hagmann, P., Goni, J., and Sporns, O. (2017). Path ensembles and a tradeoff between communication efficiency and resilience in the human connectome. Brain Struct Funct 222, 603-618.

24. Mišić, B., Sporns, O., Mcintosh, A.R., and Bassett, D.S. (2014). Communication Efficiency and Congestion of Signal Traffic in Large-Scale Brain Networks. PLoS Computational Biology,10,1(2014-1-9) 10, e1003427.

25. Dodds, P.S., Watts, D.J., and Sabel, C.F. (2003). Information exchange and the robustness of organizational networks. Proceedings of the National Academy of Sciences of the United States of America 100, 12516-12521.

26. Boguna, M., Krioukov, D., and Claffy, K. (2009). Navigability of Complex Networks. Nature Physics abs/0709.0303.

27. Guusje, Collin, Olaf, Sporns, René, Mandl, and Martijn (2014). Structural and functional aspects relating to cost and benefit of rich club organization in the human cerebral cortex. Cerebral cortex (New York, N.Y. : 1991).

28. Kaiser, M., Martin, R., Andras, P., and Young, M.P. (2007). Simulation of robustness against lesions of cortical networks. European Journal of Neuroscience 25, 3185-3192.

29. Rodrigues, F.A., and da Fontoura Costa, L. (2009). A structure-dynamic approach to cortical organization: Number of paths and accessibility. Journal of Neuroscience Methods 183, 57-62.

30. Goldberg, D.E. (1989). Genetic Algorithm in Search, Optimization, and Machine Learning, (Genetic Algorithms in Search Optimization and Machine Learning).

31. Lin, Y., Ma, J., Gu, Y., Yang, S., Li, L.M.W., and Dai, Z. (2018). Intrinsic overlapping modular organization of human brain functional networks revealed by a multiobjective evolutionary algorithm. Neuroimage 181, 430-445.

32. Ma, J., Zhang, J., Lin, Y., and Dai, Z. (2021). Cost-efficiency trade-offs of the human brain network revealed by a multiobjective evolutionary algorithm. Neuroimage 236, 118040 .

33. Weaver, B., and Wuensch, K.L. (2013). SPSS and SAS programs for comparing Pearson correlations and OLS regression coefficients. Behavior Research Methods 45, 880-895.

34. Yeo, B.T., Krienen, F.M., Sepulcre, J., Sabuncu, M.R., Lashkari, D., Hollinshead, M., Roffman, J.L., Smoller, J.W., Zollei, L., Polimeni, J.R., et al. (2011). The organization of the human cerebral cortex estimated by intrinsic functional connectivity. J Neurophysiol 106, 1125-1165. 
35. Achard, S. (2006). A resilient, low-frequency, small-world human brain functional network with highly connected association cortical hubs. Journal of Neuroscience the Official Journal of the Society for Neuroscience 26, 63.

36. van den Heuvel, M.P., and Sporns, O. (2013). An anatomical substrate for integration among functional networks in human cortex. The Journal of neuroscience : the official journal of the Society for Neuroscience 33, 14489-14500.

37. Pan, R.K., Chatterjee, N., and Sinha, S. (2010). Mesoscopic organization reveals the constraints governing Caenorhabditis elegans nervous system. In PloS one, Volume 5. p. e9240.

38. Sporns, O. (2012). The human connectome: a complex network. Ann N Y Acad 136, S28-S28.

39. Bullmore, E., and Sporns, O. (2012). The economy of brain network organization. Nature Reviews Neuroscience 13, 336-349.

40. Chan, M.Y., Park, D.C., Savalia, N.K., Petersen, S.E., and Wig, G.S. (2014). Decreased segregation of brain systems across the healthy adult lifespan. Proc Natl Acad Sci U S A 111, E4997-5006.

41. Mesulam, M.M. (2010). Large-scale neurocognitive networks and distributed processing for attention, language, and memory. Behavioral \& Brain Sciences 17, 74-76.

42. Posner, M.I., and Petersen, S.E. (1990). The Attention System Of The Human Brain. Annual Review of Neuroscience 13, 25-42.

43. Preti, M.G., and Van De Ville, D. (2019). Decoupling of brain function from structure reveals regional behavioral specialization in humans. Nature Communications 10 , 4747.

44. Baum, G.L., Cui, Z., Roalf, D.R., Ciric, R., and Satterthwaite, T.D. (2019). Development of structure-function coupling in human brain networks during youth.

45. Suárez, L., Markello, R.D., Betzel, R.F., and Misic, B. (2020). Linking Structure and Function in Macroscale Brain Networks. Trends in Cognitive Sciences 24, 302-315.

46. Preti, M.G., and Dimitri, V. Decoupling of brain function from structure reveals regional behavioral specialization in humans. Nature Communications.

47. Betzel, R.F., and Bassett, D.S. (2018). Specificity and robustness of long-distance connections in weighted, interareal connectomes. Proceedings of the National Academy of Sciences of the United States of America 115.

48. Masel, J., and Trotter, M.V. Robustness and evolvability.

49. Avena-Koenigsberger, A., Goñi, J., Betzel, R.F., van den Heuvel, M.P., Griffa, A., Hagmann, P., Thiran, J.-P., and Sporns, O. (2014). Using Pareto optimality to explore the topology and dynamics of the human connectome. Philosophical Transactions of the Royal Society B: Biological Sciences 369, 20130530.

50. Wook Yoo, S., Han, C.E., Shin, J.S., Won Seo, S., Na, D.L., Kaiser, M., Jeong, Y., and Seong, J.-K. (2015). A Network Flow-based Analysis of Cognitive Reserve in Normal Ageing and Alzheimer's Disease. Scientific Reports 5, 10057.

51. Holme, P., Kim, B.J., Yoon, C.N., and Han, S.K. (2002). Attack vulnerability of complex networks. Physical Review E Statistical Nonlinear \& Soft Matter Physics 65, 056109. 
52. Alenazi, M., and Sterbenz, J. (2015). Comprehensive comparison and accuracy of graph metrics in predicting network resilience. In 2015 11th International Conference on the Design of Reliable Communication Networks (DRCN).

53. Barthélemy, M. (2004). Betweenness Centrality in Large Complex Networks. The European Physical Journal B 38, 163-168.

54. Hannelore, A., Wim, F., Karen, C., and Daniele, M. (2016). Brain networks under attack: robustness properties and the impact of lesions. Brain A Journal of Neurology, aww194.

55. Kolb, B., Gibb, R., Robinson, and T., E. (2003). Brain Plasticity and Behavior. Current Directions in Psychological Science (Wiley-Blackwell).

56. Desmurget, M., Bonnetblanc, F., Duffau, and Hugues (2007). Contrasting acute and slow-growing lesions: a new door to brain plasticity. Brain 130, 898-914.

57. Estrada, E., Higham, D.J., and Hatano, N. (2009). Communicability betweenness in complex networks. Physica A: Statistical Mechanics and its Applications 388, 764-774.

58. Abdelnour, F., Voss, H.U., and Raj, A. (2014). Network diffusion accurately models the relationship between structural and functional brain connectivity networks. Neuroimage 90, 335-347.

59. van den Heuvel, M.P., and Sporns, O. (2013). Network hubs in the human brain. Trends Cogn Sci 17, 683-696.

60. Thomas, C., Ye, F.Q., Irfanoglu, M.O., Modi, P., Saleem, K.S., Leopold, D.A., and Pierpaoli, C. (2014). Anatomical accuracy of brain connections derived from diffusion MRI tractography is inherently limited. Proceedings of the National Academy of Sciences of the United States of America 111, 16574.

61. Bassett, D.S., Brown, J.A., Deshpande, V., Carlson, J.M., and Grafton, S.T. (2011). Conserved and variable architecture of human white matter connectivity. NeuroImage $54,1262-1279$.

62. Fair, D.A., Schlaggar, B.L., Cohen, A.L., Miezin, F.M., Dosenbach, N., Wenger, K.K., Fox, M., Snyder, A.Z., Raichle, M.E., and Petersen, S.E. (2007). A method for using blocked and event-related fMRI data to study "resting state" functional connectivity. Neuroimage 35, 396-405.

63. Cole, M.W., Bassett, D.S., Power, J.D., Braver, T.S., and Petersen, S.E. (2014). Intrinsic and Task-Evoked Network Architectures of the Human Brain. Neuron.

64. Fox, M. (2007). Intrinsic fluctuations within cortical systems account for intertrial variability in human behavior. Neuron 56, 171-184.

65. Batra, P.K., and Kant, K. (2016). An energy efficient GA based routing algorithm for two-tiered sensor networks. In 2016 1st India International Conference on Information Processing (IICIP).

66. Gupta, S.K., Kuila, P., and Jana, P.K. (2013). GAR: An Energy Efficient GA-Based Routing for Wireless Sensor Networks. 9th International Conference on Distributed Computing and Internet Technologies (ICDCIT - 2013).

67. Yue, C., and Jing, Y. (2007). Solving the Problem of the Link Optimizing and Delay-constrained Multicast Routing Based on GA. In Control Conference.

68. Lin, Q., Dai, Z., Xia, M., Han, Z., Huang, R., Gong, G., Liu, C., Bi, Y., and He, Y. 
(2015). A connectivity-based test-retest dataset of multi-modal magnetic resonance imaging in young healthy adults. Scientific Data 2, 150056.

69. Yan, C.-G., Wang, X.-D., Zuo, X.-N., and Zang, Y.-F. (2016). DPABI: Data Processing \& Analysis for (Resting-State) Brain Imaging. Neuroinformatics 14, 339-351.

70. Cui, Z., Zhong, S., Xu, P., Yong, H., and Gong, G. (2013). PANDA: a pipeline toolbox for analyzing brain diffusion images. Frontiers in Human Neuroscience 7, 42.

71. Xia, M., Wang, J., and He, Y. (2013). BrainNet Viewer: a network visualization tool for human brain connectomics. PloS one 8, e68910.

72. Leemans, A., and Jones, D.K. (2010). The B-matrix must be rotated when correcting for subject motion in DTI data. Magnetic Resonance in Medicine 61.

73. Ashburner, J., and Friston, K.J. (2005). Unified segmentation. Neuroimage 26, 839-851.

74. Tzourio-Mazoyer, N., Landeau, B., Papathanassiou, D., Crivello, F., Etard, O., Delcroix, N., Mazoyer, B., and Joliot, M. (2002). Automated anatomical labeling of activations in SPM using a macroscopic anatomical parcellation of the MNI MRI single-subject brain. Neuroimage 15, 273-289.

75. Gaolang Gong, Y.H., Luis Concha, Catherine Lebel, Donald W. Gross, Alan C. Evans, and Beaulieu, C. (2008). Mapping anatomical connectivity patterns of human cerebral cortex using in vivo diffusion tensor imaging tractography. Cerebral Cortex 19, 524-536.

76. Wang, R., Benner, T., Sorensen, A.G., and Wedeen, V.J. (2007). Diffusion Toolkit: A Software Package for Diffusion Imaging Data Processing and Tractography.

77. Sporns, O., Cammoun, L., Gigandet, X., Meuli, R., Honey, C., and Hagmann, P. (2008). Mapping the Structural Core of Human Cerebral Cortex. In Meeting Human Brain Mapping.

78. Betzel, R.F., Griffa, A., Hagmann, P., and Mišić, B. (2019). Distance-dependent consensus thresholds for generating group-representative structural brain networks. Network Neuroscience 3, 475-496.

79. Linkenkaer-Hansen, K., Nikulin, V.V., Palva, J.M., Kaila, K., and Ilmoniemi, R.J. (2004). Stimulus-induced change in long-range temporal correlations and scaling behaviour of sensorimotor oscillations. European Journal of Neuroscience 19, 203-218.

80. Valette, J., Ligneul, C., Marchadour, C., Najac, C., and Palombo, M. (2018). Brain Metabolite Diffusion from Ultra-Short to Ultra-Long Time Scales: What Do We Learn, Where Should We Go? Frontiers in Neuroscience 12.

81. Tipnis, U., Amico, E., Ventresca, M., and Goni, J. (2020). Modeling Communication Processes in the Human Connectome through Cooperative Learning. IEEE Transactions on Network Science and Engineering 7, 476-488.

82. Kumar, M., Husain, M., Upreti, N., and Gupta, D. (2010). Genetic algorithm: Review and application. Journal of Information \& Knowledge Management.

83. Vidal, T., Crainic, T.G., Gendreau, M., Lahrichi, N., and Rei, W. (2012). A Hybrid Genetic Algorithm for Multidepot and Periodic Vehicle Routing Problems. Operations Research 60, 611-624. 
84. Walters, D.C., and Sheble, G.B. (1993). Genetic algorithm solution of economic dispatch with valve point loading. IEEE Transactions on Power Systems 8, 1325-1332.

85. Deb, K., Pratap, A., Agarwal, S., and Meyarivan, T. (2002). A fast and elitist multiobjective genetic algorithm: NSGA-II. IEEE Transactions on Evolutionary Computation 6, 182-197.

86. Sharma, P., Wadhwa, A., and Komal, K. (2014). Analysis of Selection Schemes for Solving an Optimization Problem in Genetic Algorithm. International Journal of Computer Applications 93, 1-3.

87. Michalewicz, Z. (1992). Genetic Programs + Data Structures = Evolution Programs, $($ Genetic Programs + Data Structures $=$ Evolution Programs $)$. 


\section{Figure Legend}

Figure 1. Region-wise hybrid routing strategy (HYB) generated by GA. A: Structural routing efficiency obtained by HYB and the random baseline in contrast to the empirical FCN. B: Brain map of HYB. C: Modular distribution of regions using each routing strategy, where the number in the parentheses indicates the number of regions choosing the strategy. D:

Distribution of the three routing strategies used by regions in each functional module. The functional modules were derived from Yeo et al. [34] (six regions were excluded for undefined modular membership).

Figure 2. Comparison between the proposed HYB and the three classic routing strategies in terms of capturing the FCN. A: Comparison of correlations with FC at the global level. B: Comparison of correlations with FC at the modular level (only significant difference related to the HYB were annotated). C: Comparison of correlations with FC at the nodal level. D: Brain map of the 40 regions where the HYB achieved significantly higher nodal-level correlation with FC. E: Correlation between the nodal sum of structural routing efficiency achieved by the HYB and the nodal strength in the FCN. Note: only significantly positive correlations $(p<0.05)$ were considered meaningful and reported.

Figure 3. Comparison between the proposed HYB and the three classic routing strategies in terms of supporting robust communication in the SCN. A: Comparison of normalized nodal betweenness; B: Comparison of normalized edgewise betweenness; C: Comparison of relative global efficiency during targeted attack.

Figure 4. Plausibility analysis on the regional choices of routing strategies in HYB. A:

Comparison of structural degree. B: Comparison of functional participation coefficient (Pc).

C: Comparison of functional within-module degree Z-score (Z). D: Distributions of routing strategies adopted by the four types of regions classified according to the Pc-Z coordinate, 
with Pc $>0.3$ corresponding to 'high Pc' and Z > 0.9 corresponding to 'high Z'.

\section{Acknowledgement}

This work was supported by the National Natural Science Foundation of China (NSFC) (No.

61772569), the Guangdong Basic and Applied Ba- sic Research Foundation (No.

2019A1515012148, 2021A1515010844), and the Fundamental Research Funds for the

Central Universities (No. 19wkzd20, 20wkzd11).

\section{Declaration of Interests}

The authors declare no competing interests. The funders had no role in study design, data collection and analysis, decision to publish, or preparation of the manuscript.

\section{Author Contributions}

Q.L. implemented the method, performed the analyses, and wrote and edited the manuscript.

J.M. and X.C. validated the analysis and edited the manuscript. Z.D. and Y.L. designed the study, developed the method, supervised the analyses, wrote and edited the manuscript. 
A
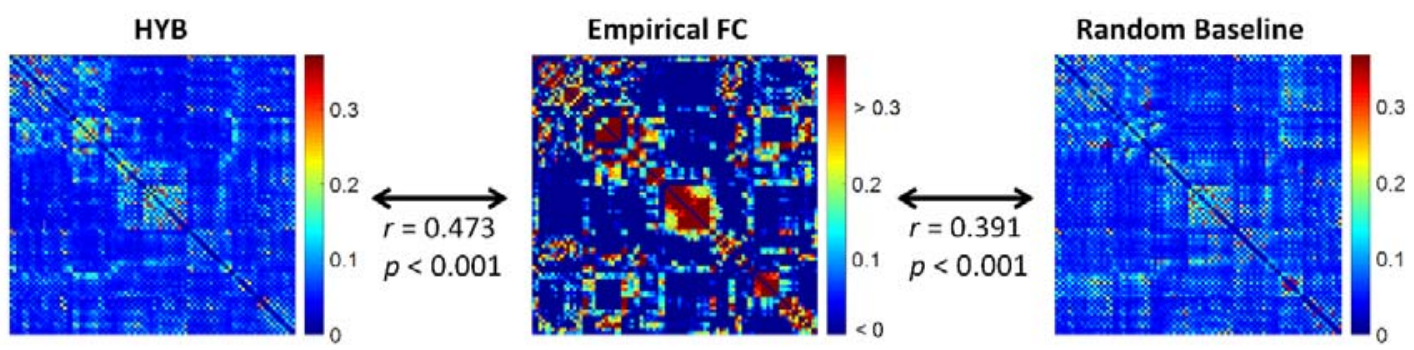

B L
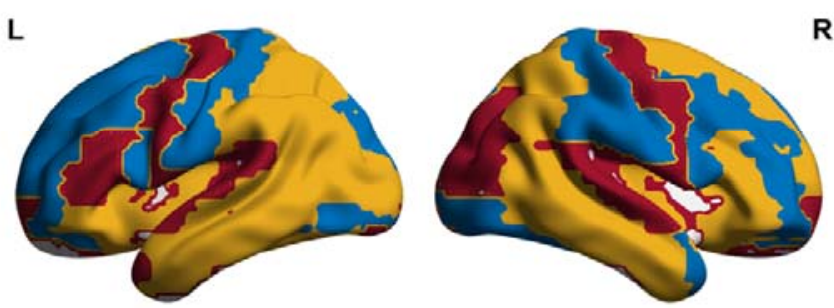

R C

$\operatorname{SP}(24)$
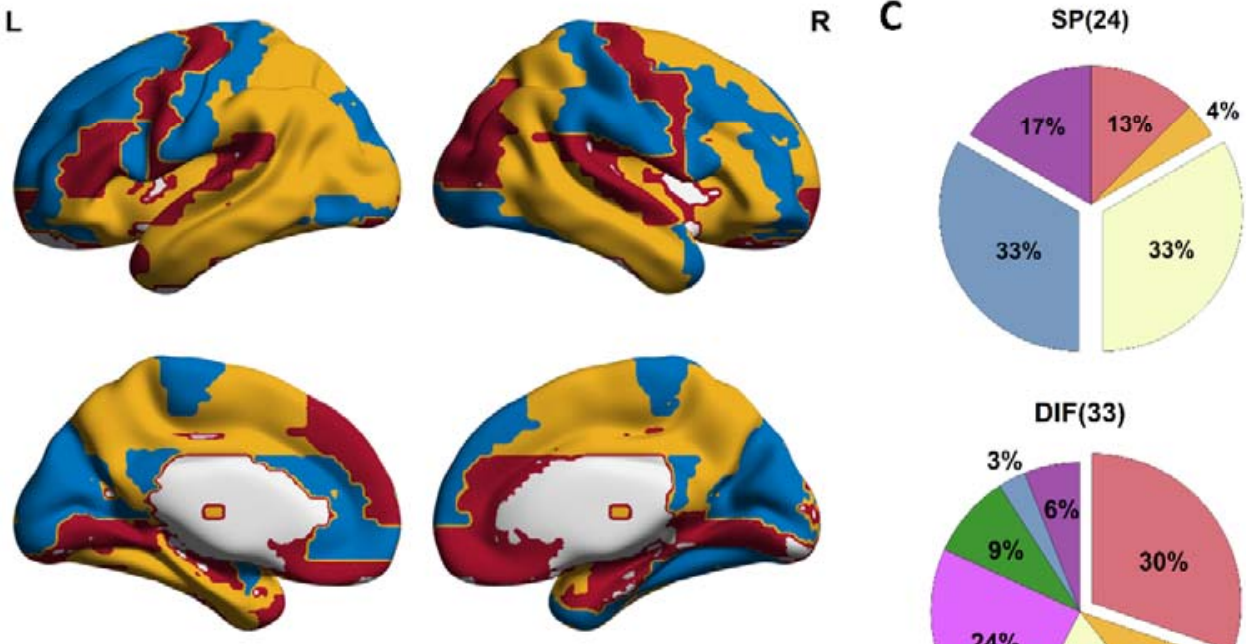

D
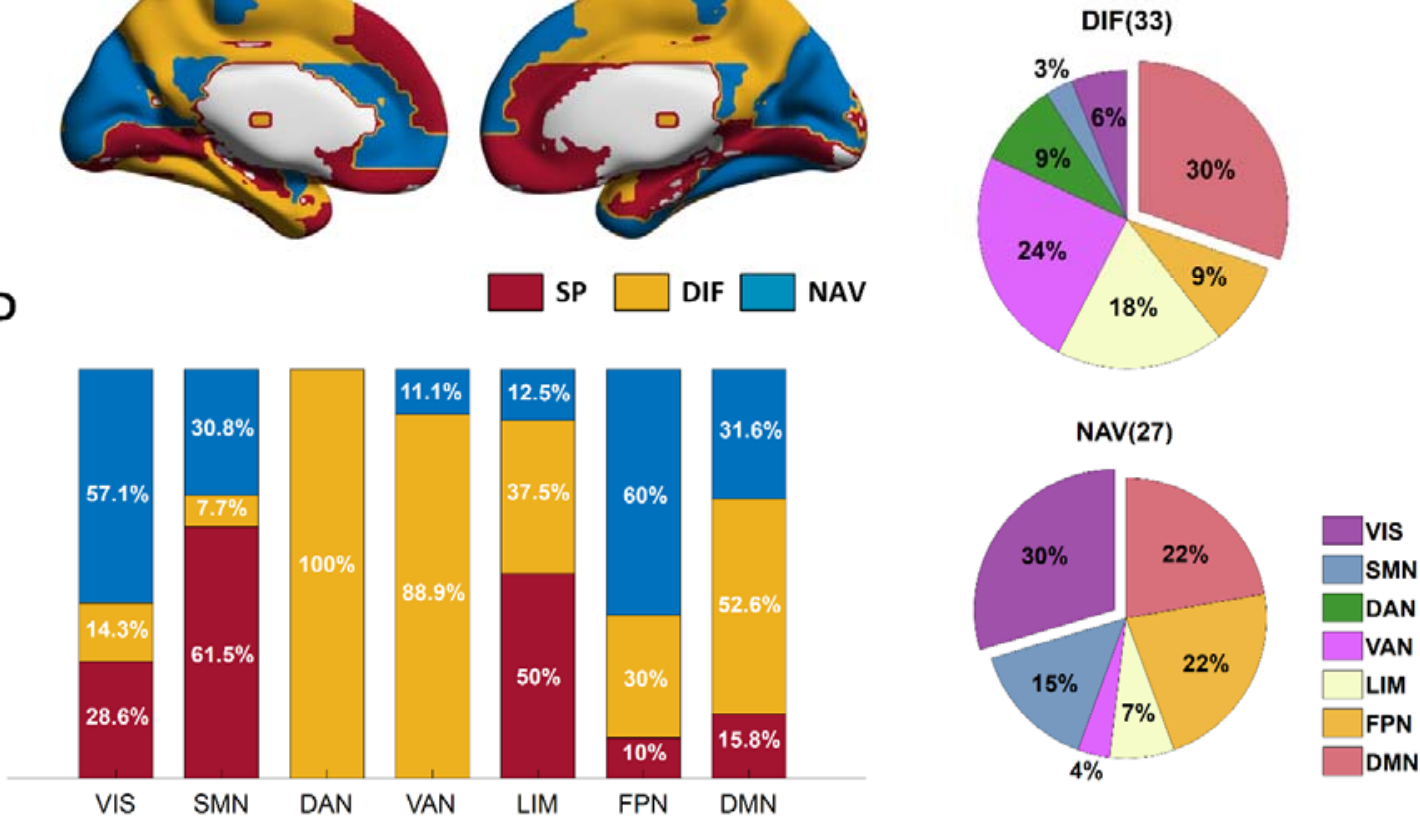

Figure 1. Region-wise hybrid routing strategy (HYB) generated by GA. A: Structural routing efficiency obtained by HYB and the random baseline in contrast to the empirical FCN. B:

Brain map of HYB. C: Modular distribution of regions using each routing strategy, where the number in the parentheses indicates the number of regions choosing the strategy. D:

Distribution of the three routing strategies used by regions in each functional module. The functional modules were derived from Yeo et al. [34] (six regions were excluded for 
undefined modular membership).

A
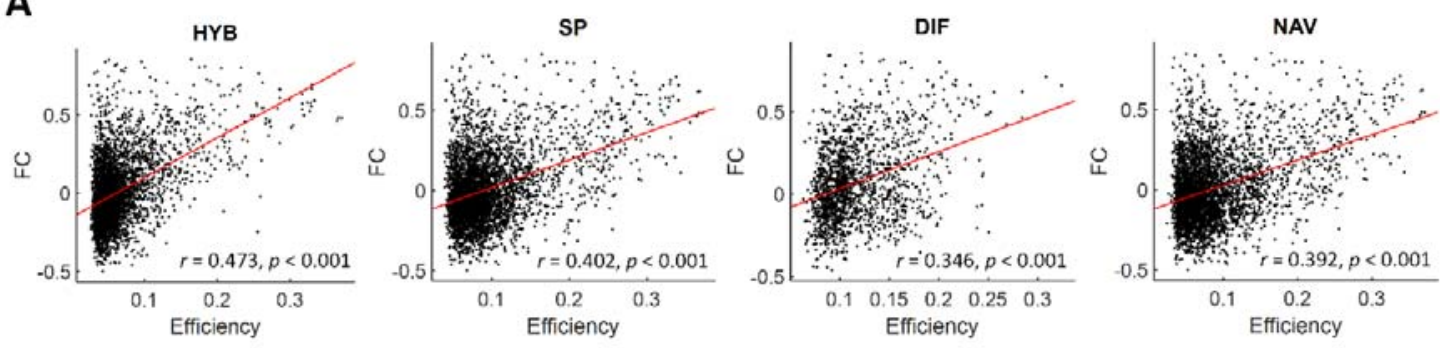

B
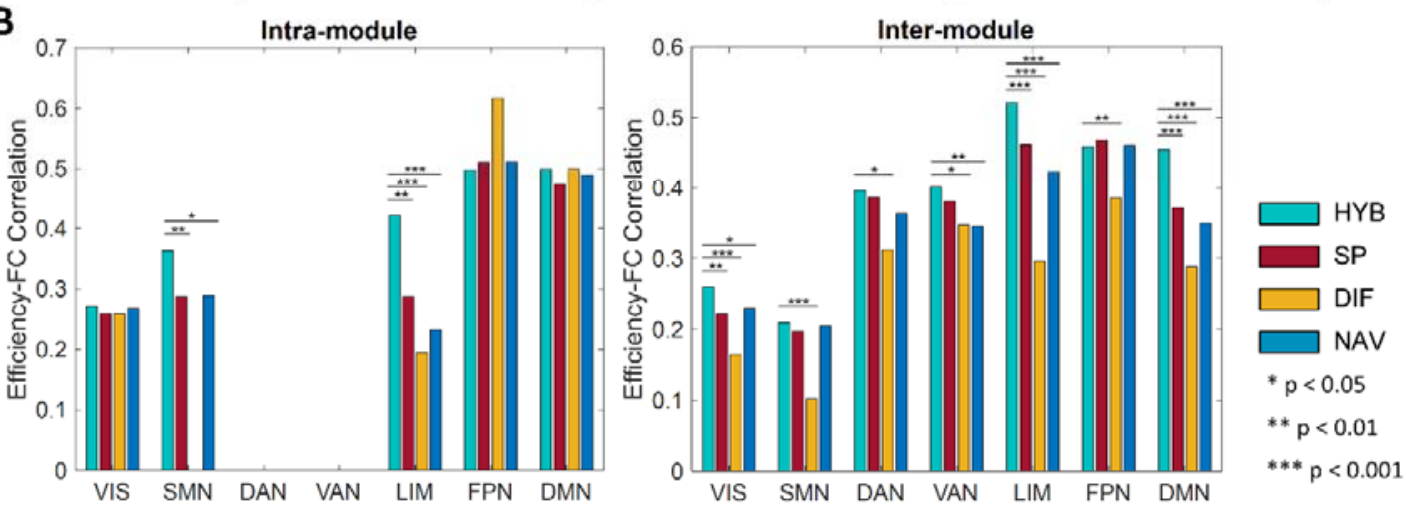

C
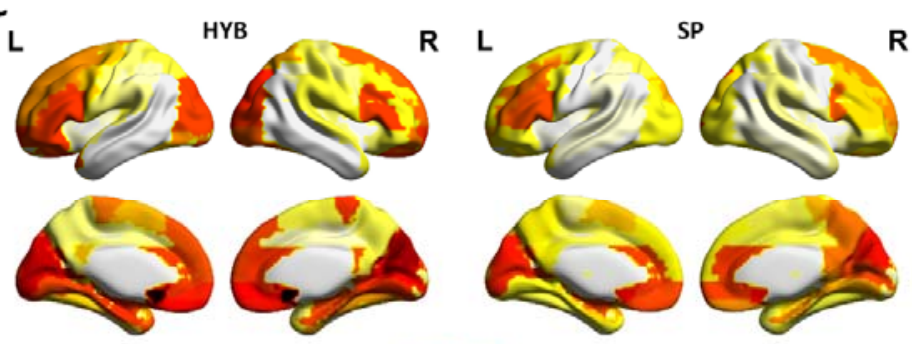

D
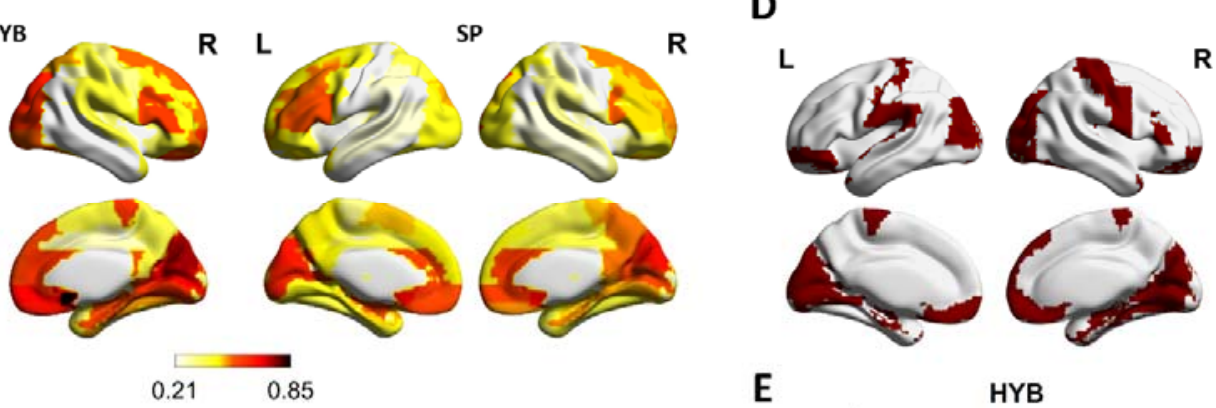

E
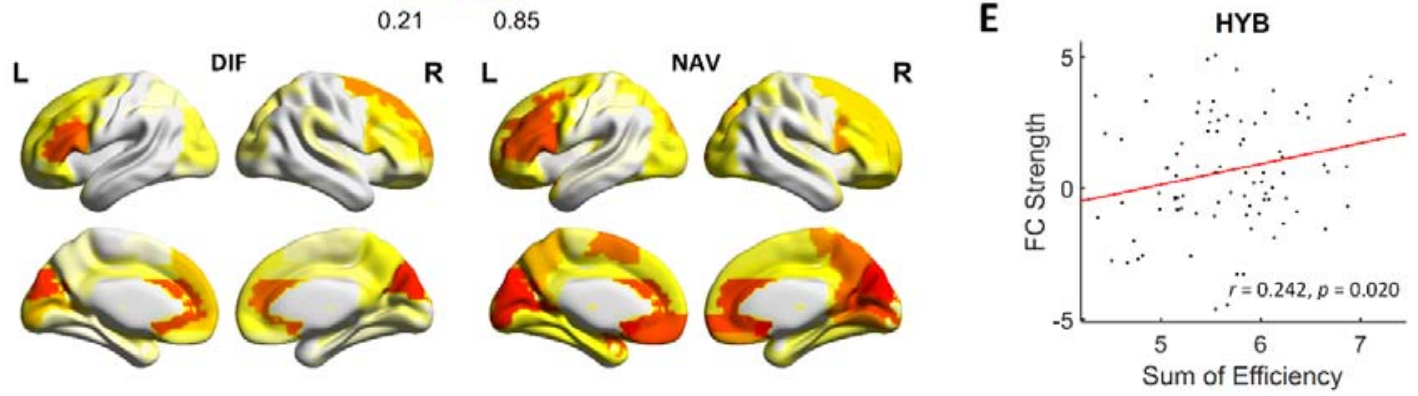

Figure 2. Comparison between the proposed HYB and the three classic routing strategies in terms of capturing the FCN. A: Comparison of correlations with FC at the global level. B: Comparison of correlations with FC at the modular level (only significant difference related 
to the HYB were annotated). C: Comparison of correlations with FC at the nodal level. D:

Brain map of the 40 regions where the HYB achieved significantly higher nodal-level

correlation with FC. E: Correlation between the nodal sum of structural routing efficiency achieved by the HYB and the nodal strength in the FCN. Note: only significantly positive correlations $(p<0.05)$ were considered meaningful results and reported.

A

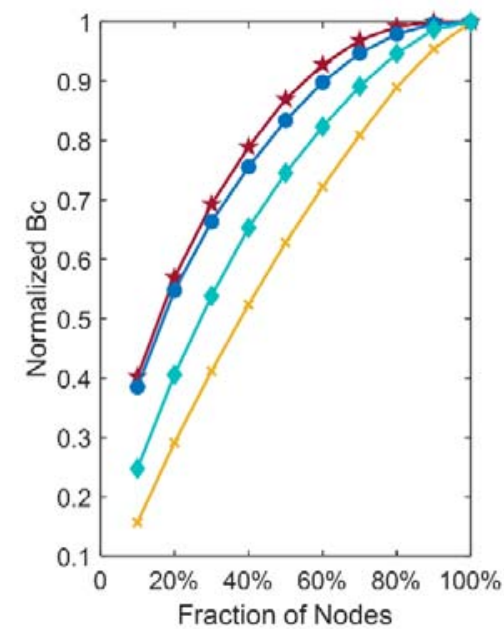

Fraction of Nodes
B

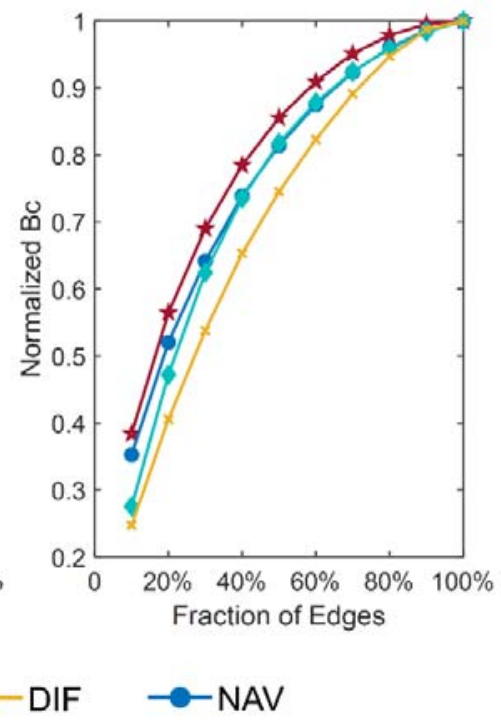

C

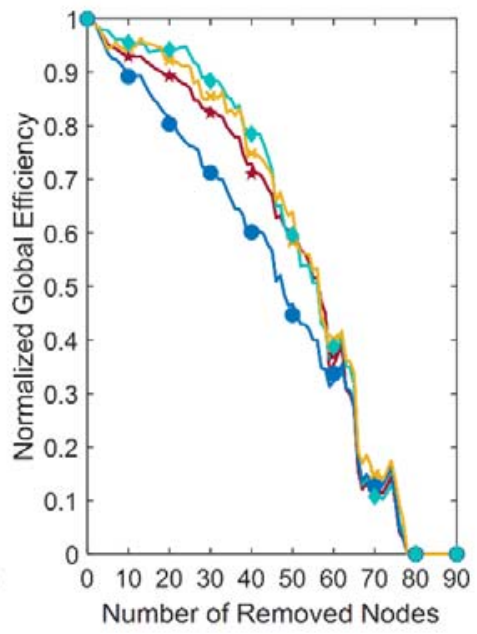

Figure 3. Comparison between the proposed HYB and the three classic routing strategies in terms of supporting robust communication in the SCN. A: Comparison of normalized nodal betweenness; B: Comparison of normalized edgewise betweenness; C: Comparison of relative global efficiency during targeted attack. 

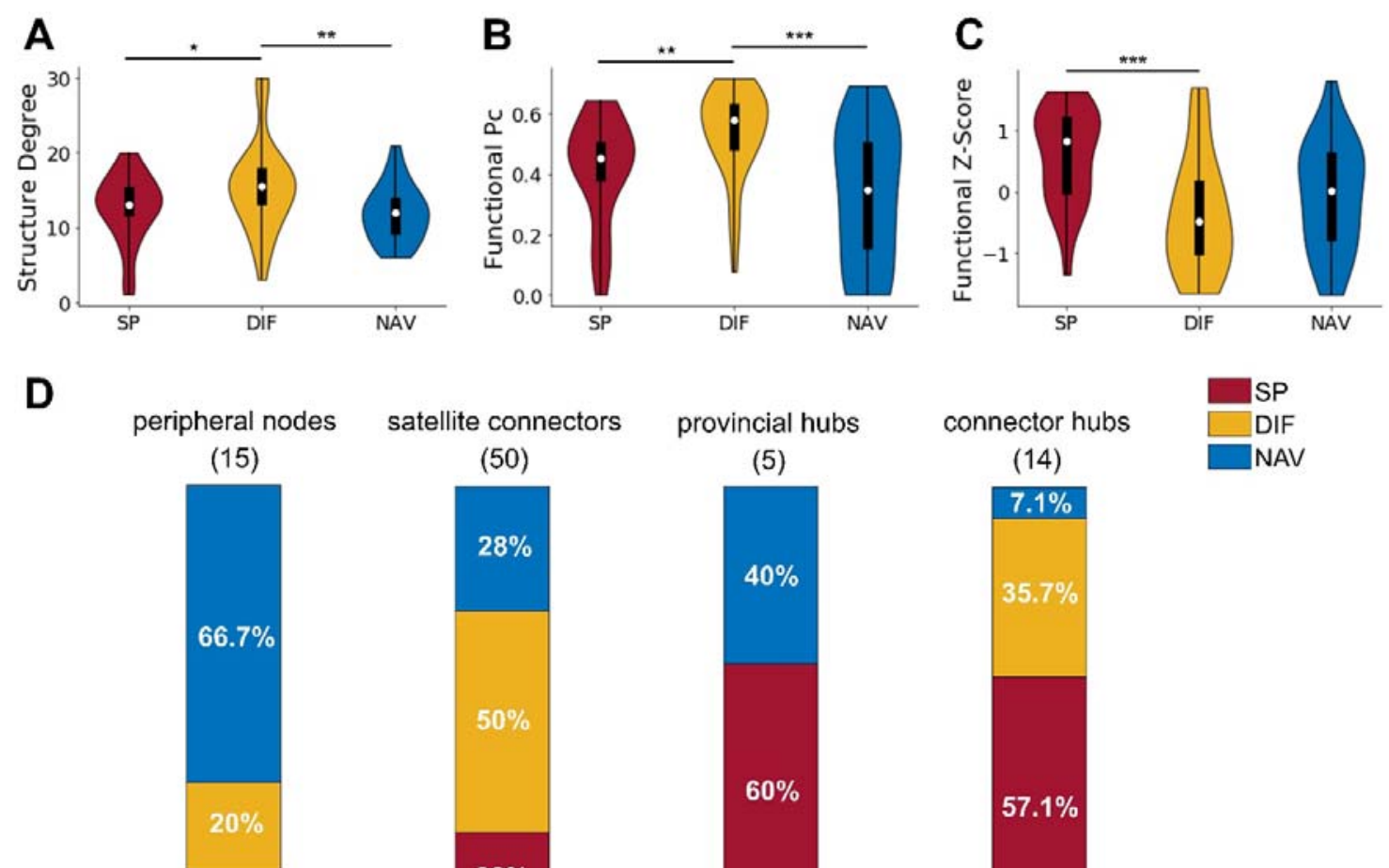

$13.3 \%$

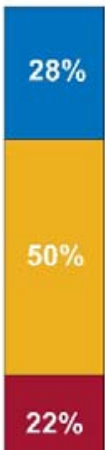

(5)

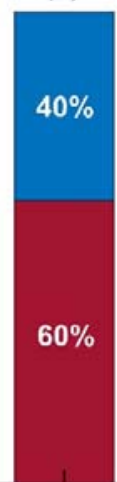

$(14)$

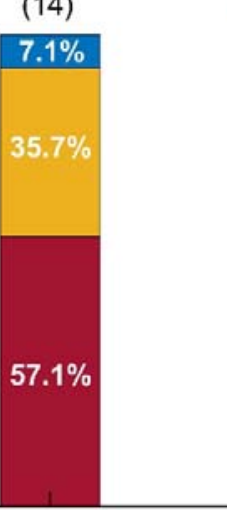

low Z/low Pc

low Z/high Pc

high Z/low Pc

high Z/high Pc

Figure 4. Plausibility analysis on the regional choices of routing strategies in HYB. A:

Comparison of structural degree. B: Comparison of functional participation coefficient (Pc).

C: Comparison of functional within-module degree Z-score (Z). D: Distributions of routing strategies adopted by the four types of regions classified according to the Pc-Z coordinate, with $\mathrm{Pc}>0.3$ corresponding to 'high Pc' and Z $>0.9$ corresponding to 'high $\mathrm{Z}$ '. 